\title{
A New Iterative Method for Solving Equilibrium Problems and Fixed Point Problems for Infinite Family of Nonexpansive Mappings
}

\author{
Shenghua Wang, ${ }^{1}$ Yeol Je Cho, ${ }^{2}$ and Xiaolong Qin ${ }^{3}$ \\ ${ }^{1}$ School of Mathematics and Physics, North China Electric Power University, Baoding 071003, China \\ ${ }^{2}$ Department of Mathematics Education and the RINS, Gyeongsang National University, \\ Chinju 660-701, Republic of Korea \\ ${ }^{3}$ Department of Mathematics, Hangzhou Normal University, Hangzhou 310036, China \\ Correspondence should be addressed to Yeol Je Cho, yjcho@gnu.ac.kr
}

Received 7 January 2010; Revised 21 May 2010; Accepted 11 July 2010

Academic Editor: Simeon Reich

Copyright (C) 2010 Shenghua Wang et al. This is an open access article distributed under the Creative Commons Attribution License, which permits unrestricted use, distribution, and reproduction in any medium, provided the original work is properly cited.

We introduce a new iterative scheme for finding a common element of the solutions sets of a finite family of equilibrium problems and fixed points sets of an infinite family of nonexpansive mappings in a Hilbert space. As an application, we solve a multiobjective optimization problem using the result of this paper.

\section{Introduction}

Let $H$ be a Hilbert space and $C$ be a nonempty, closed, and convex subset of $H$. Let $\Phi$ be a bifunction of $C \times C$ into $\mathbb{R}$, where $\mathbb{R}$ is the set of real numbers. The equilibrium problem for the bifunction $\Phi: C \times C \rightarrow \mathbb{R}$ is to find $x \in C$ such that

$$
\Phi(x, y) \geq 0, \quad \forall y \in C
$$

The set of solutions of the above inequality is denoted by $\operatorname{EP}(\Phi)$. Many problems arising from physics, optimization, and economics can reduce to finding a solution of an equilibrium problem.

In 2007, S. Takahashi and W. Takahashi [1] first introduced an iterative scheme by the viscosity approximation method for finding a common element of the solutions set of equilibrium problem and the set of fixed points of a nonexpansive mapping in a Hilbert space 
$H$ and proved a strong convergence theorem which is based on Combettes and Hirstoaga's result [2] and Wittmann's result [3]. More precisely, they obtained the following theorem.

Theorem 1.1 (see [1]). Let $C$ be a nonempty closed and convex subset of $H$. Let $\Phi: C \times C \rightarrow \mathbb{R}$ be a bifunction which satisfies the following conditions:

(A1) $\Phi(x, x)=0$ for all $x \in C$;

(A2) $\Phi$ is monotone, that is, $\Phi(x, y)+\Phi(y, x) \leq 0$ for all $x, y \in C$;

(A3) For all $x, y, z \in C$,

$$
\lim _{t \downarrow 0} \Phi(t z+(1-t) x, y) \leq \Phi(x, y)
$$

(A4) For each $x \in C, y \mapsto \Phi(x, y)$ is convex and lower semicontinuous.

Let $S: C \rightarrow H$ be a nonexpansive mapping with $\operatorname{Fix}(S) \cap \operatorname{EP}(\Phi) \neq \emptyset$, where $\operatorname{Fix}(S)$ denotes the set of fixed points of the mapping $S$, and let $f: H \rightarrow H$ be a contraction, if there exists a constant $\lambda \in(0,1)$ such that $\|f x-f y\| \leq \lambda\|x-y\|$ for all $x, y \in H$. Let $\left\{x_{n}\right\}$ and $\left\{u_{n}\right\}$ be the sequences generated by $x_{1} \in H$ and

$$
\begin{gathered}
\Phi\left(u_{n}, y\right)+\frac{1}{r_{n}}\left\langle y-u_{n}, u_{n}-x_{n}\right\rangle \geq 0, \quad \forall y \in C \\
x_{n+1}=\alpha_{n} f\left(x_{n}\right)+\left(1-\alpha_{n}\right) S u_{n}, \quad \forall n \geq 1
\end{gathered}
$$

where $\left\{\alpha_{n}\right\} \subset[0,1]$ and $\left\{r_{n}\right\} \subset(0, \infty)$ satisfy the following conditions:

$$
\begin{gathered}
\lim _{n \rightarrow \infty} \alpha_{n}=0, \quad \sum_{n=1}^{\infty} \alpha_{n}=\infty, \quad \sum_{n=1}^{\infty}\left|\alpha_{n+1}-\alpha_{n}\right|<\infty, \\
\liminf _{n \rightarrow \infty} r_{n}>0, \quad \sum_{n=1}^{\infty}\left|r_{n+1}-r_{n}\right|<\infty .
\end{gathered}
$$

Then the sequences $\left\{x_{n}\right\}$ and $\left\{u_{n}\right\}$ converge strongly to a point $z \in \operatorname{Fix}(S) \cap \operatorname{EP}(\Phi)$, where

$$
z=P_{\operatorname{Fix}(S) \cap \operatorname{EP}(\Phi)} f(z)
$$

( $P$ is the metric projection of $H$ onto $C$ and $P_{\mathrm{Fix}(S) \cap \mathrm{EP}(\Phi)} f(z)$ denotes nearest point in $\operatorname{Fix}(S) \cap \mathrm{EP}(\Phi)$ from $f(z)$ ).

Recently, many results on equilibrium problems and fixed points problems in the context of the Hilbert space and Banach space are introduced (see, e.g., [4-8]). 
Fixed Point Theory and Applications

Let $F: H \rightarrow H$ be a nonlinear mapping. The variational inequality problem corresponding to the mapping $F$ is to find a point $x^{*} \in C$ such that

$$
\left\langle F\left(x^{*}\right), x-x^{*}\right\rangle \geq 0, \quad \forall x \in C
$$

The variational inequality problem is denoted by $\mathrm{VI}(F, C)[9]$.

The mapping $F$ is called $\kappa$-Lipschitzian and $\eta$-strongly monotone if there exist constants $\kappa, \eta>0$ such that

$$
\begin{gathered}
\|F x-F y\| \leq \kappa\|x-y\|, \quad \forall x, y \in H, \\
\langle F x-F y, x-y\rangle \geq \eta\|x-y\|^{2}, \quad \forall x, y \in H,
\end{gathered}
$$

respectively. It is well known that if $F$ is strongly monotone and Lipschitzian on $C$, then $\operatorname{VI}(F, C)$ has a unique solution. An important problem is how to find a solution of $\operatorname{VI}(F, C)$. Recently, there are many results to solve the $\operatorname{VI}(F, C)$ (see, e.g., [10-14]).

Let $C$ be a nonempty closed and convex subset of a Hilbert space $H,\left\{T_{n}\right\}_{n=1}^{\infty}: H \rightarrow H$ be a countable family of nonexpansive mappings, and $\left\{\Phi_{i}\right\}_{i=1}^{m}: C \times C \rightarrow \mathbb{R}$ be $m$ bifunctions satisfying conditions (A1)-(A4) such that $\Omega=\bigcap_{n=1}^{\infty} \operatorname{Fix}\left(T_{n}\right) \cap \operatorname{EP}\left(\Phi_{1}\right) \cap \cdots \cap \operatorname{EP}\left(\Phi_{m}\right) \neq \emptyset$. Let $r_{1}, \ldots, r_{m} \in(0, \infty)$. For each $i=1, \ldots, m$, define the mapping $T_{r_{i}}: H \rightarrow C$ by

$$
T_{r_{i}}(x)=\left\{z \in C: \Phi_{i}(z, y)+\frac{1}{r_{i}}\langle y-z, z-x\rangle \geq 0, \forall y \in C\right\}, \quad \forall x \in H
$$

Lemma 2.5 (see below) shows that, for each $1 \leq i \leq m, T_{r_{i}}$ is firmly nonexpansive and hence nonexpansive and $\operatorname{Fix}\left(T_{r_{i}}\right)=\operatorname{EP}\left(\Phi_{i}\right)$. Suppose that $F: H \rightarrow H$ is a $\kappa$-Lipschitzian and $\eta$-strong monotone operator and let $\mu \in\left(0,2 \eta / \kappa^{2}\right)$. Assume that $\operatorname{VI}\left(\Phi_{i}(F, \Omega) \neq \emptyset\right.$.

In this paper, motivated and inspired by the above research results, we introduce the following iterative process for finding an element in $\Omega$ : for an arbitrary initial point $x_{1} \in H$,

$$
\begin{gathered}
z_{n}=\gamma_{1} T_{r_{1}} x_{n}+\gamma_{2} T_{r_{2}} x_{n}+\cdots+\gamma_{m} T_{r_{m}} x_{n} \\
x_{n+1}=\alpha_{n} x_{n}+\sum_{i=1}^{n}\left(\alpha_{i-1}-\alpha_{i}\right) \sigma_{n} T_{i} x_{n}+\left(1-\alpha_{n}\right)\left(1-\sigma_{n}\right) T^{\lambda_{n}} z_{n}, \quad \forall n \geq 1,
\end{gathered}
$$

where $T^{\lambda_{n}} z_{n}=z_{n}-\lambda_{n} \mu F\left(z_{n}\right), \alpha_{0}=1,\left\{\alpha_{n}\right\}_{n=1}^{\infty}$ is a strictly decreasing sequence in $(0, \alpha)$ with $0<\alpha<1,\left\{\lambda_{n}\right\}_{n=1}^{\infty} \subset(0,1),\left\{\gamma_{i}\right\}_{i=1}^{m} \subset(0,1)$ with $\sum_{i=1}^{m} \gamma_{i}=1$, and $\left\{\sigma_{n}\right\}_{n=1}^{\infty} \subset(a, b)$ with $0<a, b<$ 1 . Then we prove that the iterative process $\left\{x_{n}\right\}$ defined by (1.10) strongly converge to an element $x^{*} \in \Omega$, which is the unique solution of the variational inequality

$$
\left\langle F\left(x^{*}\right), x-x^{*}\right\rangle \geq 0, \quad \forall x \in \Omega \text {. }
$$

As an application of our main result, we solve a multiobjective optimization problem. 


\section{Preliminaries}

Let $H$ be a Hilbert space and $T$ a nonexpansive mapping of $H$ into itself such that $\operatorname{Fix}(T) \neq \emptyset$. For all $\widehat{x} \in \operatorname{Fix}(T)$ and $x \in H$, we have

$$
\begin{aligned}
\|x-\hat{x}\|^{2} & \geq\|T x-T \widehat{x}\|^{2}=\|T x-\widehat{x}\|^{2}=\|T x-x+(x-\widehat{x})\|^{2} \\
& =\|T x-x\|^{2}+\|x-\widehat{x}\|^{2}+2\langle T x-x, x-\widehat{x}\rangle
\end{aligned}
$$

and hence

$$
\|T x-x\|^{2} \leq 2\langle x-T x, x-\widehat{x}\rangle, \quad \forall \widehat{x} \in \operatorname{Fix}(T), \quad x \in H .
$$

It is well known that, for all $x, y \in H$ and $t \in[0,1]$,

$$
\|t x+(1-t) y\|^{2} \leq t\|x\|^{2}+(1-t)\|y\|^{2},
$$

which implies that

$$
\left\|\sum_{i=1}^{n} t_{i} x_{i}\right\|^{2} \leq \sum_{i=1}^{n} t_{i}\left\|x_{i}\right\|^{2}
$$

for all $\left\{x_{i}\right\}_{i=1}^{n} \subset H$ and $\left\{t_{i}\right\}_{i=1}^{n} \subset[0,1]$ with $\sum_{i=1}^{n} t_{i}=1$.

Let $C$ be a nonempty closed and convex subset of $H$ and, for any $x \in H$, there exists unique nearest point in $C$, denoted by $P_{C} x$, such that

$$
\left\|P_{C} x-x\right\| \leq\|y-x\|, \quad \forall y \in C
$$

Moreover, we have the following:

$$
z=P_{C} x \Longleftrightarrow\langle x-z, z-y\rangle \geq 0, \quad \forall y \in C .
$$

Let $I$ denote the identity operator of $H$ and let $\left\{x_{n}\right\}$ be a sequence in a Hilbert space $H$ and $x \in H$. Throughout this paper, $x_{n} \rightarrow x$ denotes that $\left\{x_{n}\right\}$ strongly converges to $x$ and $x_{n} \rightarrow x$ denotes that $\left\{x_{n}\right\}$ weakly converges to $x$.

We need the following lemmas for our main results.

Lemma 2.1 (see [15]). Let $C$ be a nonempty closed and convex subset of a Hilbert space $H$ and $T$ a nonexpansive mapping from $C$ into itself. Then $I-T$ is demiclosed at zero, that is,

$$
x_{n} \rightarrow x, \quad x_{n}-T x_{n} \longrightarrow 0 \quad \text { implies } x=T x
$$


Lemma 2.2 (see [10, Lemma 3.1(b)]). Let $H$ be a Hilbert space and $T: H \rightarrow H$ be a nonexpansive mapping. Let $F: H \rightarrow H$ be a mapping which is $\kappa$-Lipschitzian and $\eta$-strong monotone on $T(H)$. Assume that $\lambda \in(0,1)$ and $\mu \in\left(0,2 \eta / \kappa^{2}\right)$. Define a mapping $T^{\lambda}: H \rightarrow H$ by

$$
T^{\lambda} x=T x-\lambda \mu F(T x), \quad \forall x \in H .
$$

Then $\left\|T^{\lambda} x-T^{\lambda} y\right\| \leq(1-\lambda \tau)\|x-y\|$ for all $x, y \in H$, where $\tau=1-\sqrt{1-\mu\left(2 \eta-\mu \kappa^{2}\right)} \in(0,1)$.

If $T=I$, Lemma 2.2 still holds.

Lemma 2.3 (see [16]). Let $\left\{s_{n}\right\},\left\{c_{n}\right\}$ be the sequences of nonnegative real numbers and $\left\{a_{n}\right\} \subset$ $(0,1)$. Suppose that $\left\{b_{n}\right\}$ is a real number sequence such that

$$
s_{n+1} \leq\left(1-a_{n}\right) s_{n}+b_{n}+c_{n}, \quad \forall n \geq 0 .
$$

Assume that $\sum_{n=0}^{\infty} c_{n}<\infty$. Then the following results hold.

(1) If $b_{n} \leq \beta a_{n}$ for all $n \geq 0$, where $\beta \geq 0$, then $\left\{s_{n}\right\}$ is a bounded sequence.

(2) If

$$
\sum_{n=0}^{\infty} a_{n}=\infty, \quad \limsup _{n \rightarrow \infty} \frac{b_{n}}{a_{n}} \leq 0
$$

then $\lim _{n \rightarrow \infty} S_{n}=0$.

Lemma 2.4 (see [17]). Let $C$ be a nonempty closed and convex subset of a Hilbert space $H$ and $\Phi: C \times C \rightarrow \mathbb{R}$ be a bifunction which satisfies the conditions (A1)-(A4). Let $r>0$ and $x \in H$. Then there exists $z \in C$ such that

$$
\Phi(z, y)+\frac{1}{r}\langle y-z, z-x\rangle \geq 0, \quad \forall y \in C .
$$

Lemma 2.5 (see [2]). Let $H$ be a Hilbert space and $C$ be a nonempty closed and convex subset of $H$. Assume that $\Phi: C \times C \rightarrow \mathbb{R}$ satisfies the conditions (A1)-(A4). For all $r>0$ and $x \in H$, define a mapping $T_{r}: H \rightarrow C$ as follows:

$$
T_{r}(x)=\left\{z \in C: \Phi(z, y)+\frac{1}{r}\langle y-z, z-x\rangle \geq 0, \forall y \in C\right\}, \quad \forall x \in H .
$$

Then the following holds:

(1) $T_{r}$ is single-valued;

(2) $T_{r}$ is firmly nonexpansive, that is, for any $x, y \in H$,

$$
\left\|T_{r} x-T_{r} y\right\|^{2} \leq\left\langle T_{r} x-T_{r} y, x-y\right\rangle
$$

(3) $\operatorname{Fix}\left(T_{r}\right)=\operatorname{EP}(\Phi)$;

(4) $\mathrm{EP}(\Phi)$ is closed and convex. 
The following lemma is an immediate consequence of an inner product.

Lemma 2.6. Let $H$ be a real Hilbert space. Then the following identity holds:

$$
\|x+y\|^{2} \leq\|x\|^{2}+2\langle y, x+y\rangle, \quad \forall x, y \in H
$$

\section{Main Results}

First, we prove some lemmas as follows.

Lemma 3.1. The sequence $\left\{x_{n}\right\}$ generated by (1.10) is bounded.

Proof. Let $u_{i n}=T_{r_{i}} x_{n}$ for each $i=1,2, \ldots, m$. Lemma 2.5 shows that each $T_{r_{i}}$ is firmlynonexpansive and hence nonexpansive. Hence, for each $1 \leq i \leq m$ and $p \in \Omega$, we have

$$
\begin{gathered}
\left\|u_{\text {in }}-p\right\|=\left\|T_{r_{i}} x_{n}-T_{r_{i}} p\right\| \leq\left\|x_{n}-p\right\|, \quad \forall n \geq 1 \\
\left\|z_{n}-p\right\| \leq \sum_{i=1}^{m} r_{i}\left\|u_{i n}-p\right\| \leq\left\|x_{n}-p\right\|, \quad \forall n \geq 1 .
\end{gathered}
$$

By Lemma 2.2, we have

$$
\left\|T^{\lambda_{n}} x-T^{\lambda_{n}} y\right\| \leq\left(1-\lambda_{n} \tau\right)\|x-y\|, \quad \forall x, y \in H,
$$

where $\tau=1-\sqrt{1-\mu\left(2 \eta-\mu \kappa^{2}\right)} \in(0,1)$. Therefore, by (3.2) and (3.3), we obtain (note that $\left\{\alpha_{n}\right\}$ is strictly decreasing and $\left.T^{\lambda_{n}} p-p=-\lambda_{n} \mu F(p)\right)$

$$
\begin{aligned}
\left\|x_{n+1}-p\right\|= & \left\|\alpha_{n}\left(x_{n}-p\right)+\sum_{i=1}^{n}\left(\alpha_{i-1}-\alpha_{i}\right) \sigma_{n}\left(T_{i} x_{n}-p\right)+\left(1-\alpha_{n}\right)\left(1-\sigma_{n}\right)\left(T^{\lambda_{n}} z_{n}-p\right)\right\| \\
\leq & \alpha_{n}\left\|x_{n}-p\right\|+\sum_{i=1}^{n}\left(\alpha_{i-1}-\alpha_{i}\right) \sigma_{n}\left\|T_{i} x_{n}-p\right\|+\left(1-\alpha_{n}\right)\left(1-\sigma_{n}\right)\left\|T^{\lambda_{n}} z_{n}-p\right\| \\
\leq & \alpha_{n}\left\|x_{n}-p\right\|+\sum_{i=1}^{n}\left(\alpha_{i-1}-\alpha_{i}\right) \sigma_{n}\left\|x_{n}-p\right\| \\
& +\left(1-\alpha_{n}\right)\left(1-\sigma_{n}\right)\left[\left\|T^{\lambda_{n}} z_{n}-T^{\lambda_{n}} p\right\|+\left\|T^{\lambda_{n}} p-p\right\|\right] \\
\leq & \alpha_{n}\left\|x_{n}-p\right\|+\sum_{i=1}^{n}\left(\alpha_{i-1}-\alpha_{i}\right) \sigma_{n}\left\|x_{n}-p\right\| \\
& +\left(1-\alpha_{n}\right)\left(1-\sigma_{n}\right)\left[\left(1-\lambda_{n} \tau\right)\left\|z_{n}-p\right\|+\lambda_{n} \mu\|F(p)\|\right]
\end{aligned}
$$


Fixed Point Theory and Applications

$$
\begin{aligned}
\leq & \alpha_{n}\left\|x_{n}-p\right\|+\sum_{i=1}^{n}\left(\alpha_{i-1}-\alpha_{i}\right) \sigma_{n}\left\|x_{n}-p\right\| \\
& +\left(1-\alpha_{n}\right)\left(1-\sigma_{n}\right)\left[\left(1-\lambda_{n} \tau\right)\left\|x_{n}-p\right\|+\lambda_{n} \mu\|F(p)\|\right] \\
= & \left(1-\left(1-\alpha_{n}\right)\left(1-\sigma_{n}\right) \lambda_{n} \tau\right)\left\|x_{n}-p\right\|+\left(1-\alpha_{n}\right)\left(1-\sigma_{n}\right) \lambda_{n} \mu\|F(p)\| .
\end{aligned}
$$

By induction, we obtain $\left\|x_{n+1}\right\| \leq \max \left\{\left\|x_{1}-p\right\|,(\mu / \tau)\|F(p)\|\right\}$. Hence $\left\{x_{n}\right\}$ is bounded and so are $\left\{z_{n}\right\}$ and $\left\{u_{i n}\right\}$ for each $i=1,2, \ldots, m$. Since $F$ is $\kappa$-Lipschitzian, we have

$$
\begin{aligned}
\left\|F\left(z_{n}\right)\right\| & \leq\left\|F\left(z_{n}\right)-F(p)\right\|+\|F(p)\| \\
& \leq \kappa\left\|z_{n}-p\right\|+\|F(p)\| \leq \kappa\left\|z_{n}\right\|+\kappa\|p\|+\|F(p)\|,
\end{aligned}
$$

which shows that $\left\{F\left(z_{n}\right)\right\}$ is bounded. This completes the proof.

Lemma 3.2. If the following conditions hold:

$$
\sum_{n=1}^{\infty} \lambda_{n}=\infty, \quad \sum_{n=1}^{\infty}\left|\lambda_{n}-\lambda_{n+1}\right|<\infty, \quad \sum_{n=1}^{\infty}\left|\sigma_{n}-\sigma_{n+1}\right|<\infty,
$$

then $\lim _{n \rightarrow \infty}\left\|x_{n+1}-x_{n}\right\|=0$.

Proof. For each $i=1,2, \ldots, m$, since each $T_{r_{i}}$ is nonexpansive, we have

$$
\left\|u_{i n-1}-u_{i n}\right\|=\left\|T_{r_{i}} x_{n-1}-T_{r_{i}} x_{n}\right\| \leq\left\|x_{n-1}-x_{n}\right\|, \quad \forall n \geq 1 .
$$

By (3.7), we have

$$
\begin{aligned}
\left\|z_{n}-z_{n-1}\right\| & =\left\|\gamma_{1}\left(u_{1 n}-u_{1 n-1}\right)+\gamma_{2}\left(u_{2 n}-u_{2 n-1}\right)+\cdots+\gamma_{m}\left(u_{m n}-u_{m n-1}\right)\right\| \\
& \leq \sum_{i=1}^{m} \gamma_{i}\left\|u_{i n}-u_{i n-1}\right\| \leq \sum_{i=1}^{m} \gamma_{i}\left\|x_{n}-x_{n-1}\right\| \\
& =\left\|x_{n-1}-x_{n}\right\|, \quad \forall n \geq 1 .
\end{aligned}
$$

By the definition of the iterative sequence (1.10), we have

$$
\begin{aligned}
x_{n+1}-x_{n}= & \alpha_{n}\left(x_{n}-x_{n-1}\right)+\alpha_{n} x_{n-1}+\sum_{i=1}^{n}\left(\alpha_{i-1}-\alpha_{i}\right) \sigma_{n}\left(T_{i} x_{n}-T_{i} x_{n-1}\right) \\
& +\sum_{i=1}^{n}\left(\alpha_{i-1}-\alpha_{i}\right) \sigma_{n} T_{i} x_{n-1}+\left(1-\alpha_{n}\right)\left(1-\sigma_{n}\right)\left(T^{\lambda_{n}} z_{n}-T^{\lambda_{n}} z_{n-1}\right) \\
& +\left(1-\alpha_{n}\right)\left(1-\sigma_{n}\right) T^{\lambda_{n}} z_{n-1}-\alpha_{n-1} x_{n-1}-\sum_{i=1}^{n-1}\left(\alpha_{i-1}-\alpha_{i}\right) \sigma_{n-1} T_{i} x_{n-1} \\
& -\left(1-\alpha_{n-1}\right)\left(1-\sigma_{n-1}\right) T^{\lambda_{n-1}} z_{n-1}
\end{aligned}
$$




$$
\begin{aligned}
= & \alpha_{n}\left(x_{n}-x_{n-1}\right)+\left(\alpha_{n}-\alpha_{n-1}\right) x_{n-1}+\sum_{i=1}^{n}\left(\alpha_{i-1}-\alpha_{i}\right) \sigma_{n}\left(T_{i} x_{n}-T_{i} x_{n-1}\right) \\
& +\left(1-\alpha_{n}\right)\left(1-\sigma_{n}\right)\left(T^{\lambda_{n}} z_{n}-T^{\lambda_{n}} z_{n-1}\right)+\sum_{i=1}^{n}\left(\alpha_{i-1}-\alpha_{i}\right) \sigma_{n} T_{i} x_{n-1} \\
& -\sum_{i=1}^{n-1}\left(\alpha_{i-1}-\alpha_{i}\right) \sigma_{n-1} T_{i} x_{n-1}+\left(1-\alpha_{n}\right)\left(1-\sigma_{n}\right) T^{\lambda_{n}} z_{n-1} \\
& -\left(1-\alpha_{n-1}\right)\left(1-\sigma_{n-1}\right) T^{\lambda_{n-1}} z_{n-1} \\
= & \alpha_{n}\left(x_{n}-x_{n-1}\right)+\left(\alpha_{n}-\alpha_{n-1}\right) x_{n-1}+\sum_{i=1}^{n}\left(\alpha_{i-1}-\alpha_{i}\right) \sigma_{n}\left(T_{i} x_{n}-T_{i} x_{n-1}\right) \\
& +\left(1-\alpha_{n}\right)\left(1-\sigma_{n}\right)\left(T^{\lambda_{n}} z_{n}-T^{\lambda_{n}} z_{n-1}\right)+\sum_{i=1}^{n-1}\left(\alpha_{i-1}-\alpha_{i}\right)\left(\sigma_{n}-\sigma_{n-1}\right) T_{i} x_{n-1} \\
& +\left(\alpha_{n-1}-\alpha_{n}\right) \sigma_{n} T_{n} x_{n-1}+\left[\left(\alpha_{n-1}-\alpha_{n}\right)\left(1-\sigma_{n}\right)+\left(\sigma_{n-1}-\sigma_{n}\right)\left(1-\alpha_{n-1}\right)\right] z_{n-1} \\
& +\left\{\left(1-\alpha_{n-1}\right)\left(1-\sigma_{n-1}\right)\left(\lambda_{n-1}-\lambda_{n}\right)\right. \\
& \left.\quad-\left[\left(\alpha_{n-1}-\alpha_{n}\right)\left(1-\sigma_{n}\right)+\left(\sigma_{n-1}-\sigma_{n}\right)\left(1-\alpha_{n-1}\right)\right] \lambda_{n}\right\} \mu F\left(z_{n-1}\right)
\end{aligned}
$$

and hence

$$
\begin{aligned}
\left\|x_{n+1}-x_{n}\right\| \leq & \alpha_{n}\left\|x_{n}-x_{n-1}\right\|+\left(\alpha_{n-1}-\alpha_{n}\right)\left\|x_{n-1}\right\|+\sum_{i=1}^{n}\left(\alpha_{i-1}-\alpha_{i}\right) \sigma_{n}\left\|x_{n}-x_{n-1}\right\| \\
& +\left(1-\alpha_{n}\right)\left(1-\sigma_{n}\right)\left(1-\lambda_{n} \tau\right)\left\|z_{n}-z_{n-1}\right\|+\sum_{i=1}^{n-1}\left(\alpha_{i-1}-\alpha_{i}\right)\left|\sigma_{n}-\sigma_{n-1}\right|\left\|T_{i} x_{n-1}\right\| \\
& +\left(\alpha_{n-1}-\alpha_{n}\right)\left\|T_{n} x_{n-1}\right\|+\left[\left(\alpha_{n-1}-\alpha_{n}\right)+\left|\sigma_{n-1}-\sigma_{n}\right|\right]\left\|z_{n-1}\right\| \\
& +\left[\left|\lambda_{n-1}-\lambda_{n}\right|+\left(\alpha_{n-1}-\alpha_{n}\right)+\left|\sigma_{n-1}-\sigma_{n}\right|\right] \mu\left\|F\left(z_{n-1}\right)\right\| \\
= & \alpha_{n}\left\|x_{n}-x_{n-1}\right\|+\sum_{i=1}^{n}\left(\alpha_{i-1}-\alpha_{i}\right) \sigma_{n}\left\|x_{n}-x_{n-1}\right\| \\
& +\left(1-\alpha_{n}\right)\left(1-\sigma_{n}\right)\left(1-\lambda_{n} \tau\right)\left\|z_{n}-z_{n-1}\right\| \\
& +\left(\alpha_{n-1}-\alpha_{n}\right)\left[\left\|x_{n-1}\right\|+\left\|T_{n} x_{n-1}\right\|+\left\|z_{n-1}\right\|+\mu\left\|F\left(z_{n-1}\right)\right\|\right] \\
& +\sum_{i=1}^{n-1}\left(\alpha_{i-1}-\alpha_{i}\right)\left|\sigma_{n}-\sigma_{n-1}\right|\left\|T_{i} x_{n-1}\right\|+\left|\sigma_{n-1}-\sigma_{n}\right|\left(\left\|z_{n-1}\right\|+\mu\left\|F\left(z_{n-1}\right)\right\|\right) \\
& +\left|\lambda_{n-1}-\lambda_{n}\right| \mu\left\|F\left(z_{n-1}\right)\right\| .
\end{aligned}
$$


It follows from (3.8) and (3.10) that

$$
\begin{aligned}
\left\|x_{n+1}-x_{n}\right\| \leq & \alpha_{n}\left\|x_{n}-x_{n-1}\right\|+\sum_{i=1}^{n}\left(\alpha_{i-1}-\alpha_{i}\right) \sigma_{n}\left\|x_{n}-x_{n-1}\right\| \\
& +\left(1-\alpha_{n}\right)\left(1-\sigma_{n}\right)\left(1-\lambda_{n} \tau\right)\left\|x_{n-1}-x_{n}\right\| \\
& +\left(\alpha_{n-1}-\alpha_{n}\right)\left[\left\|x_{n-1}\right\|+\left\|T_{n} x_{n-1}\right\|+\left\|z_{n-1}\right\|+\mu\left\|F\left(z_{n-1}\right)\right\|\right] \\
& +\sum_{i=1}^{n-1}\left(\alpha_{i-1}-\alpha_{i}\right)\left|\sigma_{n}-\sigma_{n-1}\right|\left\|T_{i} x_{n-1}\right\|+\left|\sigma_{n-1}-\sigma_{n}\right|\left(\left\|z_{n-1}\right\|+\mu\left\|F\left(z_{n-1}\right)\right\|\right) \\
& +\left|\lambda_{n-1}-\lambda_{n}\right| \mu\left\|F\left(z_{n-1}\right)\right\| \\
\leq & \left(1-\left(1-\alpha_{n}\right)\left(1-\sigma_{n}\right) \lambda_{n} \tau\right)\left\|x_{n}-x_{n-1}\right\|+\left(\alpha_{n-1}-\alpha_{n}\right)(3+\mu) M \\
& +\left|\sigma_{n}-\sigma_{n-1}\right|(2+\mu) M+\left|\lambda_{n-1}-\lambda_{n}\right| \mu M \\
\leq & \left(1-(1-\alpha)(1-b) \lambda_{n} \tau\right)\left\|x_{n}-x_{n-1}\right\|+\left(\alpha_{n-1}-\alpha_{n}\right)(3+\mu) M \\
& +\left|\sigma_{n}-\sigma_{n-1}\right|(2+\mu) M+\left|\lambda_{n-1}-\lambda_{n}\right| \mu M,
\end{aligned}
$$

where $M=\max \left\{\sup _{n \geq 1}\left\|x_{n}\right\|, \sup _{n \geq 1}\left\|z_{n}\right\|, \sup _{i \geq 1, n \geq 1}\left\|T_{i} x_{n}\right\|, \sup _{n \geq 1}\left\|F\left(z_{n}\right)\right\|\right\}$. Since $\left\{\alpha_{n}\right\}$ is strictly decreasing, we have $\sum_{n=2}^{\infty}\left(\alpha_{n-1}-\alpha_{n}\right)=\alpha_{1}<\infty$. Further, from the assumptions, it follows that

$$
\sum_{n=2}^{\infty}\left\{\left(\alpha_{n-1}-\alpha_{n}\right)(3+\mu) M+\left|\sigma_{n}-\sigma_{n-1}\right|(2+\mu) M+\left|\lambda_{n-1}-\lambda_{n}\right| \mu M\right\}<\infty .
$$

Therefore, by Lemma 2.3, we have $\lim _{n \rightarrow \infty}\left\|x_{n+1}-x_{n}\right\|=0$. This completes the proof.

Lemma 3.3. If the following conditions hold:

$$
\lim _{n \rightarrow \infty} \lambda_{n}=0, \quad \sum_{n=1}^{\infty} \lambda_{n}=\infty, \quad \sum_{n=1}^{\infty}\left|\lambda_{n}-\lambda_{n+1}\right|<\infty, \quad \sum_{n=1}^{\infty}\left|\sigma_{n}-\sigma_{n+1}\right|<\infty,
$$

then $\lim _{n \rightarrow \infty}\left\|x_{n}-u_{\text {in }}\right\|=0$ for each $i=1,2, \ldots, m$.

Proof. For any $p \in \Omega$ and $i=1,2, \ldots, m$, it follows from Lemma 2.5(2) that

$$
\begin{aligned}
\left\|u_{\text {in }}-p\right\|^{2} & =\left\|T_{r_{i}} x_{n}-T_{r_{i}} p\right\|^{2} \leq\left\langle T_{r_{i}} x_{n}-T_{r_{i}} p, x_{n}-p\right\rangle=\left\langle u_{\text {in }}-p, x_{n}-p\right\rangle \\
& =\frac{1}{2}\left(\left\|u_{\text {in }}-p\right\|^{2}+\left\|x_{n}-p\right\|^{2}-\left\|u_{\text {in }}-x_{n}\right\|^{2}\right),
\end{aligned}
$$


and hence $\left\|u_{\text {in }}-p\right\|^{2} \leq\left\|x_{n}-p\right\|^{2}-\left\|u_{\text {in }}-x_{n}\right\|^{2}$. Further, we have

$$
\begin{aligned}
\left\|z_{n}-p\right\|^{2} & =\left\|\sum_{i=1}^{m} r_{i}\left(u_{i n}-p\right)\right\|^{2} \leq \sum_{i=1}^{m} r_{i}\left\|u_{i n}-p\right\|^{2} \\
& \leq \sum_{i=1}^{m} r_{i}\left(\left\|x_{n}-p\right\|^{2}-\left\|u_{i n}-x_{n}\right\|^{2}\right) \\
& =\left\|x_{n}-p\right\|^{2}-\sum_{i=1}^{m} r_{i}\left\|u_{i n}-x_{n}\right\|^{2}, \quad \forall n \geq 1 .
\end{aligned}
$$

Therefore, from (2.4) and (3.3), we have

$$
\begin{aligned}
& \left\|x_{n+1}-p\right\|^{2}=\left\|\alpha_{n}\left(x_{n}-p\right)+\sum_{i=1}^{n}\left(\alpha_{i-1}-\alpha_{i}\right) \sigma_{n}\left(T_{i} x_{n}-p\right)+\left(1-\alpha_{n}\right)\left(1-\sigma_{n}\right)\left(T^{\lambda_{n}} z_{n}-p\right)\right\|^{2} \\
& \leq \alpha_{n}\left\|x_{n}-p\right\|^{2}+\sum_{i=1}^{n}\left(\alpha_{i-1}-\alpha_{i}\right) \sigma_{n}\left\|T_{i} x_{n}-p\right\|^{2}+\left(1-\alpha_{n}\right)\left(1-\sigma_{n}\right)\left\|T^{\lambda_{n}} z_{n}-p\right\|^{2} \\
& \leq \alpha_{n}\left\|x_{n}-p\right\|^{2}+\left(1-\alpha_{n}\right) \sigma_{n}\left\|x_{n}-p\right\|^{2} \\
& +\left(1-\alpha_{n}\right)\left(1-\sigma_{n}\right)\left[\left\|T^{\lambda_{n}} z_{n}-T^{\lambda_{n}} p\right\|+\left\|T^{\lambda_{n}} p-p\right\|\right]^{2} \\
& \leq \alpha_{n}\left\|x_{n}-p\right\|^{2}+\left(1-\alpha_{n}\right) \sigma_{n}\left\|x_{n}-p\right\|^{2} \\
& +\left(1-\alpha_{n}\right)\left(1-\sigma_{n}\right)\left[\left(1-\lambda_{n} \tau\right)\left\|z_{n}-p\right\|+\lambda_{n} \mu\|F(p)\|\right]^{2} \\
& \leq \alpha_{n}\left\|x_{n}-p\right\|^{2}+\left(1-\alpha_{n}\right) \sigma_{n}\left\|x_{n}-p\right\|^{2}+\left(1-\alpha_{n}\right)\left(1-\sigma_{n}\right) \\
& \times\left[\left(1-\lambda_{n} \tau\right)\left\|z_{n}-p\right\|^{2}+2 \lambda_{n}\left(1-\lambda_{n} \tau\right) \mu\left\|z_{n}-p\right\|\|F(p)\|+\lambda_{n} \mu^{2}\|F(p)\|^{2}\right] \\
& \leq \alpha_{n}\left\|x_{n}-p\right\|^{2}+\left(1-\alpha_{n}\right) \sigma_{n}\left\|x_{n}-p\right\|^{2}+\left(1-\alpha_{n}\right)\left(1-\sigma_{n}\right) \\
& \times\left[\left(1-\lambda_{n} \tau\right)\left(\left\|x_{n}-p\right\|^{2}-\sum_{i=1}^{m} \gamma_{i}\left\|u_{i n}-x_{n}\right\|^{2}\right)\right. \\
& \left.+2 \lambda_{n}\left(1-\lambda_{n} \tau\right) \mu\left\|z_{n}-p\right\|\|F(p)\|+\lambda_{n} \mu^{2}\|F(p)\|^{2}\right] \\
& =\left(1-\left(1-\alpha_{n}\right)\left(1-\sigma_{n}\right) \lambda_{n} \tau\right)\left\|x_{n}-p\right\|^{2}-\left(1-\alpha_{n}\right)\left(1-\sigma_{n}\right)\left(1-\lambda_{n} \tau\right) \sum_{i=1}^{m} \gamma_{i}\left\|u_{i n}-x_{n}\right\|^{2} \\
& +2 \lambda_{n} \mu\left(1-\alpha_{n}\right)\left(1-\sigma_{n}\right)\left(1-\lambda_{n} \tau\right)\left\|z_{n}-p\right\|\|F(p)\|+\left(1-\alpha_{n}\right)\left(1-\sigma_{n}\right) \lambda_{n} \mu^{2}\|F(p)\|^{2}
\end{aligned}
$$


Fixed Point Theory and Applications

$$
\begin{aligned}
\leq & \left\|x_{n}-p\right\|^{2}-\left(1-\alpha_{n}\right)\left(1-\sigma_{n}\right)\left(1-\lambda_{n} \tau\right) \sum_{i=1}^{m} \gamma_{i}\left\|u_{i n}-x_{n}\right\|^{2} \\
& +\left(1-\alpha_{n}\right)\left(1-\sigma_{n}\right) \lambda_{n} \mu^{2}\|F(p)\|^{2} \\
& +2 \lambda_{n} \mu\left(1-\alpha_{n}\right)\left(1-\sigma_{n}\right)\left(1-\lambda_{n} \tau\right)\left\|z_{n}-p\right\|\|F(p)\| .
\end{aligned}
$$

It follows that

$$
\begin{aligned}
& \gamma_{i}\left(1-\alpha_{n}\right)\left(1-\sigma_{n}\right)\left(1-\lambda_{n} \tau\right)\left\|u_{i n}-x_{n}\right\|^{2} \\
& \quad \leq\left(\left\|x_{n}-p\right\|+\left\|x_{n+1}-p\right\|\right)\left\|x_{n}-x_{n+1}\right\|+\lambda_{n}\left[\mu^{2}\|F(p)\|^{2}+2 \mu\left\|z_{n}-p\right\|\|F(p)\|\right]
\end{aligned}
$$

for each $i=1,2, \ldots, m$. Note that $0<\gamma_{i}<1$ for $i=1,2, \ldots, m$. From the assumptions, Lemma 3.2, and the previous inequality, we conclude that $\left\|u_{i n}-x_{n}\right\| \rightarrow 0$ as $n \rightarrow \infty$ for each $i=1,2, \ldots, m$. Further, we have

$$
\left\|z_{n}-x_{n}\right\| \leq \sum_{i=1}^{m} \gamma_{i}\left\|u_{i n}-x_{n}\right\| \longrightarrow 0 \quad(n \longrightarrow \infty)
$$

This completes the proof.

Lemma 3.4. If the following conditions hold:

$$
\lim _{n \rightarrow \infty} \lambda_{n}=0, \quad \sum_{n=1}^{\infty} \lambda_{n}=\infty, \quad \sum_{n=1}^{\infty}\left|\lambda_{n}-\lambda_{n+1}\right|<\infty, \quad \sum_{n=1}^{\infty}\left|\sigma_{n}-\sigma_{n+1}\right|<\infty,
$$

then $\lim _{n \rightarrow \infty}\left\|x_{n}-T_{i} x_{n}\right\|=0$ for all $i \geq 1$.

Proof. By the definition of the iterative sequence (1.10), we have

$$
x_{n+1}+\sum_{i=1}^{n}\left(\alpha_{i-1}-\alpha_{i}\right) \sigma_{n}\left(x_{n}-T_{i} x_{n}\right)-\left(1-\alpha_{n}\right) \sigma_{n} x_{n}=\alpha_{n} x_{n}+\left(1-\alpha_{n}\right)\left(1-\sigma_{n}\right) T^{\lambda_{n}} z_{n}
$$

that is,

$$
\begin{aligned}
\sum_{i=1}^{n}\left(\alpha_{i-1}-\alpha_{i}\right) \sigma_{n}\left(x_{n}-T_{i} x_{n}\right) & =x_{n}-x_{n+1}-x_{n}+\alpha_{n} x_{n}+\left(1-\alpha_{n}\right) \sigma_{n} x_{n}+\left(1-\alpha_{n}\right)\left(1-\sigma_{n}\right) T^{\lambda_{n}} z_{n} \\
& =x_{n}-x_{n+1}+\left(1-\alpha_{n}\right)\left(\sigma_{n}-1\right) x_{n}+\left(1-\alpha_{n}\right)\left(1-\sigma_{n}\right) T^{\lambda_{n}} z_{n} \\
& =x_{n}-x_{n+1}+\left(1-\alpha_{n}\right)\left(1-\sigma_{n}\right)\left(T^{\lambda_{n}} z_{n}-x_{n}\right) .
\end{aligned}
$$


Hence, for any $p \in \Omega$, we get

$$
\sum_{i=1}^{n}\left(\alpha_{i-1}-\alpha_{i}\right) \sigma_{n}\left\langle x_{n}-T_{i} x_{n}, x_{n}-p\right\rangle=\left(1-\alpha_{n}\right)\left(1-\sigma_{n}\right)\left\langle T^{\lambda_{n}} z_{n}-x_{n}, x_{n}-p\right\rangle+\left\langle x_{n}-x_{n+1}, x_{n}-p\right\rangle .
$$

Since each $T_{i}$ is nonexpansive, by (2.2), we have

$$
\left\|T_{i} x_{n}-x_{n}\right\|^{2} \leq 2\left\langle x_{n}-T_{i} x_{n}, x_{n}-p\right\rangle .
$$

Hence, combining this inequality with (3.22), we get

$$
\frac{1}{2} \sum_{i=1}^{n}\left(\alpha_{i-1}-\alpha_{i}\right) \sigma_{n}\left\|T_{i} x_{n}-x_{n}\right\|^{2} \leq\left(1-\alpha_{n}\right)\left(1-\sigma_{n}\right)\left\langle T^{\lambda_{n}} z_{n}-x_{n}, x_{n}-p\right\rangle+\left\langle x_{n}-x_{n+1}, x_{n}-p\right\rangle,
$$

which implies that (note that $\left\{\alpha_{n}\right\}$ is a strictly decreasing sequence)

$$
\begin{aligned}
\left\|T_{i} x_{n}-x_{n}\right\|^{2} & \leq \frac{2\left(1-\alpha_{n}\right)\left(1-\sigma_{n}\right)}{\left(\alpha_{i-1}-\alpha_{i}\right) \sigma_{n}}\left\langle T^{\lambda_{n}} z_{n}-x_{n}, x_{n}-p\right\rangle+\frac{2}{\left(\alpha_{i-1}-\alpha_{i}\right) \sigma_{n}}\left\langle x_{n}-x_{n+1}, x_{n}-p\right\rangle \\
& \leq \frac{2\left(1-\alpha_{n}\right)\left(1-\sigma_{n}\right)}{\left(\alpha_{i-1}-\alpha_{i}\right) \sigma_{n}}\left\|T^{\lambda_{n}} z_{n}-x_{n}\right\|\left\|x_{n}-p\right\|+\frac{2}{\left(\alpha_{i-1}-\alpha_{i}\right) \sigma_{n}}\left\|x_{n}-x_{n+1}\right\|\left\|x_{n}-p\right\| .
\end{aligned}
$$

From Lemma 3.3, $\lim _{n \rightarrow \infty} \lambda_{n}=0$, and the inequality

$$
\left\|T^{\lambda_{n}} z_{n}-x_{n}\right\| \leq\left\|z_{n}-x_{n}\right\|+\lambda_{n} \mu\left\|F\left(z_{n}\right)\right\|
$$

we obtain

$$
\lim _{n \rightarrow \infty}\left\|T^{\lambda_{n}} z_{n}-x_{n}\right\|=0
$$

Therefore, from Lemma 3.2, (3.25), and (3.27), it follows that

$$
\lim _{n \rightarrow \infty}\left\|T_{i} x_{n}-x_{n}\right\|=0, \quad \forall i \geq 1
$$

This completes the proof. 
Fixed Point Theory and Applications

Next we prove the main results of this paper.

Theorem 3.5. Assume that the following conditions hold:

$$
\begin{gathered}
\lim _{n \rightarrow \infty} \lambda_{n}=0, \quad \sum_{n=1}^{\infty} \lambda_{n}=\infty, \quad \sum_{n=1}^{\infty}\left|\lambda_{n}-\lambda_{n+1}\right|<\infty, \\
\sum_{n=1}^{\infty}\left|\gamma_{n}-\gamma_{n+1}\right|<\infty, \quad \sum_{n=1}^{\infty}\left|\sigma_{n}-\sigma_{n+1}\right|<\infty .
\end{gathered}
$$

Then the sequence $\left\{x_{n}\right\}$ generated by (1.10) converges strongly to an element in $\Omega$, which is the unique solution of the variational inequality $\operatorname{VI}(F, \Omega)$.

Proof. Since $\operatorname{VI}(F, \Omega) \neq \emptyset$, we can select an element $x^{*} \in \operatorname{VI}(F, \Omega)$, which implies that

$$
\left\langle F\left(x^{*}\right), x^{*}-x\right\rangle \geq 0, \quad \forall x \in \Omega .
$$

First, we prove that

$$
\limsup _{n \rightarrow \infty}\left\langle-F\left(x^{*}\right), x_{n+1}-x^{*}\right\rangle \leq 0
$$

Since $\left\{x_{n}\right\}$ is bounded, there exists a subsequence $\left\{x_{n_{j}}\right\}$ of $\left\{x_{n}\right\}$ such that

$$
\limsup _{n \rightarrow \infty}\left\langle-F\left(x^{*}\right), x_{n}-x^{*}\right\rangle=\lim _{j \rightarrow \infty}\left\langle-F\left(x^{*}\right), x_{n_{j}}-x^{*}\right\rangle .
$$

Without loss of generality, we may further assume that $x_{n_{j}}-\hat{x}$ for some $\hat{x} \in H$. From Lemmas 3.4 and 2.1, we get $\hat{x} \in \operatorname{Fix}\left(T_{n}\right)$ for all $n \geq 1$. Hence we have $\hat{x} \in \bigcap_{n=1}^{\infty} \operatorname{Fix}\left(T_{n}\right)$. It follows from Lemma 2.5 that each $T_{r_{i}}$ is firmly nonexpansive and hence nonexpansive. Lemma 3.3 shows that $\left\|T_{r_{i}} x_{n}-x_{n}\right\| \rightarrow 0$ as $n \rightarrow \infty$. Therefore, from Lemma 2.1, it follows that $\hat{x} \in \operatorname{Fix}\left(T_{r_{i}}\right)$ for each $i=1, \ldots, m$, which shows that $\widehat{x} \in \bigcap_{i=1}^{m} \operatorname{Fix}\left(T_{r_{i}}\right)$. Lemma 2.5 shows that $\operatorname{Fix}\left(T_{r_{i}}\right)=\operatorname{EP}\left(\Phi_{i}\right)$ for each $i=1, \ldots, m$. Hence $\hat{x} \in \bigcap_{i=1}^{m} \operatorname{EP}\left(\Phi_{i}\right)$. By using the above argument, we conclude that

$$
\widehat{x} \in \Omega=\bigcap_{n=1}^{\infty} \operatorname{Fix}\left(T_{n}\right) \cap \operatorname{EP}\left(\Phi_{1}\right) \cap \cdots \cap \operatorname{EP}\left(\Phi_{m}\right) .
$$

Noting that $x^{*}$ is a solution of the $\operatorname{VI}(F, \Omega)$, we obtain

$$
\limsup _{n \rightarrow \infty}\left\langle-F\left(x^{*}\right), x_{n}-x^{*}\right\rangle=\left\langle-F\left(x^{*}\right), \widehat{x}-x^{*}\right\rangle \leq 0 .
$$


It follows from Lemma 2.6 that

$$
\begin{aligned}
\left\|x_{n+1}-x^{*}\right\|^{2} & \|\left[\alpha_{n}\left(x_{n}-x^{*}\right)+\sum_{i=1}^{n}\left(\alpha_{i-1}-\alpha_{n}\right) \sigma_{n}\left(T_{i} x_{n}-x^{*}\right)+\left(1-\alpha_{n}\right)\left(1-\sigma_{n}\right)\left(T^{\lambda_{n}} z_{n}-T^{\lambda_{n}} x^{*}\right)\right] \\
= & \quad\left(1-\alpha_{n}\right)\left(1-\sigma_{n}\right)\left(T^{\lambda_{n}} x^{*}-x^{*}\right) \|^{2} \\
& \left\|\alpha_{n}\left(x_{n}-x^{*}\right)+\sum_{i=1}^{n}\left(\alpha_{i-1}-\alpha_{n}\right) \sigma_{n}\left(T_{i} x_{n}-x^{*}\right)+\left(1-\alpha_{n}\right)\left(1-\sigma_{n}\right)\left(T^{\lambda_{n}} z_{n}-T^{\lambda_{n}} x^{*}\right)\right\|^{2} \\
& +2\left(1-\alpha_{n}\right)\left(1-\sigma_{n}\right)\left\langle T^{\lambda_{n}} x^{*}-x^{*}, x_{n+1}-x^{*}\right\rangle \\
\leq & \alpha_{n}\left\|x_{n}-x^{*}\right\|^{2}+\sum_{i=1}^{n}\left(\alpha_{i-1}-\alpha_{n}\right) \sigma_{n}\left\|T_{i} x_{n}-x^{*}\right\|^{2}+\left(1-\alpha_{n}\right)\left(1-\sigma_{n}\right)\left\|T^{\lambda_{n}} z_{n}-T^{\lambda_{n}} x^{*}\right\|^{2} \\
& +2\left(1-\alpha_{n}\right)\left(1-\sigma_{n}\right) \lambda_{n} \mu\left\langle-F\left(x^{*}\right), x_{n+1}-x^{*}\right\rangle \\
\leq & \alpha_{n}\left\|x_{n}-x^{*}\right\|^{2}+\sum_{i=1}^{n}\left(\alpha_{i-1}-\alpha_{n}\right) \sigma_{n}\left\|x_{n}-x^{*}\right\|^{2}+\left(1-\alpha_{n}\right)\left(1-\sigma_{n}\right)\left(1-\lambda_{n} \tau\right)\left\|z_{n}-x^{*}\right\|^{2} \\
& +2\left(1-\alpha_{n}\right)\left(1-\sigma_{n}\right) \lambda_{n} \mu\left\langle-F\left(x^{*}\right), x_{n+1}-x^{*}\right\rangle \\
= & \alpha_{n}\left\|x_{n}-x^{*}\right\|^{2}+\left(1-\alpha_{n}\right) \sigma_{n}\left\|x_{n}-x^{*}\right\|^{2}+\left(1-\alpha_{n}\right)\left(1-\sigma_{n}\right)\left(1-\lambda_{n} \tau\right)\left\|z_{n}-x^{*}\right\|^{2} \\
& +2\left(1-\alpha_{n}\right)\left(1-\sigma_{n}\right) \lambda_{n} \mu\left\langle-F\left(x^{*}\right), x_{n+1}-x^{*}\right\rangle \\
\leq & \alpha_{n}\left\|x_{n}-x^{*}\right\|^{2}+\left(1-\alpha_{n}\right) \sigma_{n}\left\|x_{n}-x^{*}\right\|^{2}+\left(1-\alpha_{n}\right)\left(1-\sigma_{n}\right)\left(1-\lambda_{n} \tau\right)\left\|x_{n}-x^{*}\right\|^{2} \\
& +2\left(1-\alpha_{n}\right)\left(1-\sigma_{n}\right) \lambda_{n} \mu\left\langle-F\left(x^{*}\right), x_{n+1}-x^{*}\right\rangle \\
= & \left(1-\left(1-\alpha_{n}\right)\left(1-\sigma_{n}\right) \lambda_{n} \tau\right)\left\|x_{n}-x^{*}\right\|+2\left(1-\alpha_{n}\right)\left(1-\sigma_{n}\right) \lambda_{n} \mu\left\langle-F\left(x^{*}\right), x_{n+1}-x^{*}\right\rangle .
\end{aligned}
$$

Let $a_{n}=\left(1-\alpha_{n}\right)\left(1-\sigma_{n}\right) \lambda_{n} \tau$ and $b_{n}=2\left(1-\alpha_{n}\right)\left(1-\sigma_{n}\right) \lambda_{n} \mu\left\langle-F\left(x^{*}\right), x_{n+1}-x^{*}\right\rangle$ for all $n \geq 1$. Then, from the assumptions and (3.31), we have

$$
0<a_{n}<1, \quad \sum_{n=1}^{\infty} a_{n}=\infty, \quad \limsup _{n \rightarrow \infty} \frac{b_{n}}{a_{n}}=0 .
$$

Therefore, by applying Lemma 2.3 to (3.35), we conclude that the sequence $\left\{x_{n}\right\}$ strongly converges to a point $x^{*}$.

In order to prove the uniqueness of solution of the $\operatorname{VI}(F, \Omega)$, we assume that $u^{*}$ is another solution of $\operatorname{VI}(F, \Omega)$. Similarly, we can conclude that $\left\{x_{n}\right\}$ converges strongly to a point $u^{*}$. Hence $x^{*}=u^{*}$, that is, $x^{*}$ is the unique solution of $\operatorname{VI}(F, \Omega)$. This completes the proof. 
As direct consequences of Theorem 3.5, we obtain the following corollaries.

Corollary 3.6. Let $C$ be a nonempty closed and convex subset of a Hilbert space $H$. For each $i=$ $1,2, \ldots, m$ let $\Phi_{i}: C \times C \rightarrow \mathbb{R}$ be $m$ bifunctions which satisfy conditions (A1)-(A4) such that $\bigcap_{i=1}^{m} \operatorname{EP}\left(\Phi_{i}\right) \neq \emptyset$. Let $\mu \in(0,2)$, and let $\left\{\alpha_{n}\right\}_{n=1}^{\infty} \subset(0, \alpha)$ be a strictly decreasing sequence with $0<\alpha<$ $1,\left\{\lambda_{n}\right\}_{n=1}^{\infty} \subset(0,1),\left\{\gamma_{i}\right\}_{i=1}^{m} \subset(0,1)$ with $\sum_{i=1}^{m} \gamma_{i}=1, r_{1}, r_{2}, \ldots, r_{m} \in(0, \infty)$, and $\left\{\sigma_{n}\right\}_{n=1}^{n} \subset(a, b)$ with $0<a, b<1$. For an arbitrary initial $x_{1} \in H$, define the iterative sequence $\left\{x_{n}\right\}$ by

$$
\begin{gathered}
z_{n}=\gamma_{1} T_{r_{1}} x_{n}+\gamma_{2} T_{r_{2}} x_{n}+\cdots+\gamma_{m} T_{r_{m}} x_{n} \\
x_{n+1}=\left(\alpha_{n}+\left(1-\alpha_{n}\right) \sigma_{n}\right) x_{n}+\left(1-\alpha_{n}\right)\left(1-\sigma_{n}\right)\left(1-\lambda_{n} \mu\right) z_{n}, \quad \forall n \geq 1 .
\end{gathered}
$$

If the following conditions hold:

$$
\lim _{n \rightarrow \infty} \lambda_{n}=0, \quad \sum_{n=1}^{\infty} \lambda_{n}=\infty, \quad \sum_{n=1}^{\infty}\left|\lambda_{n}-\lambda_{n+1}\right|<\infty, \quad \sum_{n=1}^{\infty}\left|\sigma_{n}-\sigma_{n+1}\right|<\infty,
$$

then the sequence $\left\{x_{n}\right\}$ converges strongly to an element $x^{*} \in \bigcap_{i=1}^{m} \operatorname{EP}\left(\Phi_{i}\right)$.

Proof. Put $F=I$ and $T_{i}=I$ for each $i \geq 1$ in Theorem 3.5. Then we know that $F$ is 1 Lipschitzian and 1-strongly monotone, $\sum_{i=1}^{n}\left(\alpha_{i-1}-\alpha_{i}\right) T_{i} x_{n}=\left(1-\alpha_{n}\right) x_{n}$ and $T^{\lambda_{n}} z_{n}=\left(1-\lambda_{n} \mu\right) z_{n}$. Therefore, by Theorem 3.5, we conclude the desired result.

Corollary 3.7. Let $C$ be a nonempty closed and convex subset of a Hilbert space $H$. Let $\left\{T_{i}\right\}_{i=1}^{\infty}$ be a countable family of nonexpansive mappings of $H$ such that $C=\bigcap_{i=1}^{\infty} \operatorname{Fix}\left(T_{i}\right)$ and $F: H \rightarrow H$ an operator which is $\kappa$-Lipschitzian and $\eta$-strong monotone on $H$. Let $\mu \in\left(0,2 \eta / \kappa^{2}\right)$. Assume that $\mathrm{VI}(F, C) \neq \emptyset$. Let $\left\{\alpha_{n}\right\}_{n=1}^{\infty} \subset(0, \alpha)$ with $0<\alpha<1$ be a strictly decreasing sequence, $\left\{\lambda_{n}\right\}_{n=1}^{\infty} \subset(0,1)$ and $\left\{\sigma_{n}\right\}_{n=1}^{\infty} \subset(a, b)$ with $0<a, b<1$. For an arbitrary initial $x_{1} \in H$, define the iterative sequence $\left\{x_{n}\right\}$ by

$$
x_{n+1}=\alpha_{n} x_{n}+\sum_{i=1}^{n}\left(\alpha_{i-1}-\alpha_{i}\right) \sigma_{n} T_{i} x_{n}+\left(1-\alpha_{n}\right)\left(1-\sigma_{n}\right)\left(P_{C} x_{n}-\lambda_{n} \mu F\left(P_{C} x_{n}\right)\right), \quad \forall n \geq 1,
$$

where $\alpha_{0}=1$. If the following conditions hold:

$$
\lim _{n \rightarrow \infty} \lambda_{n}=0, \quad \sum_{n=1}^{\infty} \lambda_{n}=\infty, \quad \sum_{n=1}^{\infty}\left|\lambda_{n}-\lambda_{n+1}\right|<\infty, \quad \sum_{n=1}^{\infty}\left|\sigma_{n}-\sigma_{n+1}\right|<\infty,
$$

then the sequence $\left\{x_{n}\right\}$ strongly converges to an element $x^{*} \in C$, which is the unique solution of the variational inequality

$$
\left\langle F\left(x^{*}\right), x-x^{*}\right\rangle \geq 0, \quad \forall x \in C
$$

Proof. Put $\Phi_{i}(x, y)=0$ for each $i=1,2, \ldots, m$ and $x, y \in C$. Set $r_{1}=r_{2}=\cdots=r_{m}=1$ in Theorem 3.5. Then, by (2.6), we have $T_{r_{1}} x_{n}=T_{r_{2}} x_{n}=\cdots=T_{r_{m}} x_{n}=P_{C} x_{n}$. Therefore, by Theorem 3.5, we conclude the desired result. 
Remark 3.8. (1) Recently, many authors have studied the iteration sequences for infinite family of nonexpansive mappings. But our iterative sequence (1.10) is very different from others because we do not use $W$-mapping generated by the infinite family of nonexpansive mappings and we have no any restriction with the infinite family of nonlinear mappings.

(2) We do not use Suzuki's lemma [18] for obtaining the result that $\lim _{n \rightarrow \infty} \| x_{n+1}-$ $x_{n} \|=0$. However, many authors have used Suzuki's lemma [18] for obtaining the result that $\lim _{n \rightarrow \infty}\left\|x_{n+1}-x_{n}\right\|=0$ in the process of studying the similar algorithms. For example, see $[5,19,20]$ and so on.

\section{Application}

In this section, we study a kind of multiobjective optimization problem based on the result of this paper. That is, we give an iterative sequence which solves the following multiobjective optimization problem with nonempty set of solutions:

$$
\begin{gathered}
\min h_{1}(x), \\
\min h_{2}(x), \\
x \in C,
\end{gathered}
$$

where $h_{1}(x)$ and $h_{2}(x)$ are both convex and lower semicontinuous functions defined on a nonempty closed and convex subset of $C$ of a Hilbert space $H$. We denote by $A$ the set of solutions of (4.1) and assume that $A \neq \emptyset$.

We denote the sets of solutions of the following two optimization problems by $A_{1}$ and $A_{2}$, respectively,

$$
\begin{gathered}
\min h_{1}(x) \\
x \in C, \\
\min h_{2}(x) \\
x \in C .
\end{gathered}
$$

Obviously, if we find a solution $x \in A_{1} \cap A_{2}$, then one must have $x \in A$.

Now, let $\Phi_{1}$ and $\Phi_{2}$ be two bifunctions from $C \times C$ to $\mathbb{R}$ defined by $\Phi_{1}(x, y)=$ $h_{1}(y)-h_{1}(x)$ and $\Phi_{2}(x, y)=h_{2}(y)-h_{2}(x)$, respectively. It is easy to see that $\operatorname{EP}\left(\Phi_{1}\right)=A_{1}$ and $\operatorname{EP}\left(\Phi_{2}\right)=A_{2}$, where $\operatorname{EP}\left(\Phi_{i}\right)$ denotes the set of solutions of the equilibrium problem:

$$
\Phi_{i}(x, y) \geq 0, \quad \forall y \in C, i=1,2,
$$

respectively. In addition, it is easy to see that $\Phi_{1}$ and $\Phi_{2}$ satisfy the conditions (A1)-(A4). Therefore, by setting $m=2$ in Corollary 3.6, we know that, for any initial guess $x_{1} \in H$,

$$
\begin{gathered}
h_{1}(y)-h_{1}\left(u_{1 n}\right)+\frac{1}{r_{1 n}}\left\langle y-u_{1 n}, u_{1 n}-x_{n}\right\rangle \geq 0, \quad \forall y \in C, \\
h_{2}(y)-h_{2}\left(u_{2 n}\right)+\frac{1}{r_{2 n}}\left\langle y-u_{2 n}, u_{2 n}-x_{n}\right\rangle \geq 0, \quad \forall y \in C, \\
z_{n}=\gamma_{1} u_{1 n}+\left(1-\gamma_{1}\right) u_{2 n}, \\
x_{n+1}=\left(\alpha_{n}+\left(1-\alpha_{n}\right) \sigma_{n}\right) x_{n}+\left(1-\alpha_{n}\right)\left(1-\sigma_{n}\right)\left(1-\lambda_{n} \mu\right) z_{n}, \quad \forall n \geq 1 .
\end{gathered}
$$


By Corollary 3.6, we know that the sequence $\left\{x_{n}\right\}$ converges strongly to a solution $x^{*} \in$ $\mathrm{EP}\left(\Phi_{1}\right) \cap \operatorname{EP}\left(\Phi_{2}\right)=A_{1} \cap A_{2}$, which is a solution of the multiobjective optimization problem (4.1).

\section{Acknowledgment}

This work was supported by the Korea Research Foundation Grant funded by the Korean Government (KRF-2008-313-C00050).

\section{References}

[1] S. Takahashi and W. Takahashi, "Viscosity approximation methods for equilibrium problems and fixed point problems in Hilbert spaces," Journal of Mathematical Analysis and Applications, vol. 331, no. 1, pp. 506-515, 2007.

[2] P. L. Combettes and S. A. Hirstoaga, "Equilibrium programming in Hilbert spaces," Journal of Nonlinear and Convex Analysis, vol. 6, no. 1, pp. 117-136, 2005.

[3] R. Wittmann, "Approximation of fixed points of nonexpansive mappings," Archiv der Mathematik, vol. 58, no. 5, pp. 486-491, 1992.

[4] L. C. Ceng, S. Schaible, and J. C. Yao, "Implicit iteration scheme with perturbed mapping for equilibrium problems and fixed point problems of finitely many nonexpansive mappings," Journal of Optimization Theory and Applications, vol. 139, no. 2, pp. 403-418, 2008.

[5] L. C. Ceng, A. Petruşel, and J. C. Yao, "Iterative approaches to solving equilibrium problems and fixed point problems of infinitely many nonexpansive mappings," Journal of Optimization Theory and Applications, vol. 143, no. 1, pp. 37-58, 2009.

[6] S.-S. Chang, Y. J. Cho, and J. K. Kim, "Approximation methods of solutions for equilibrium problem in Hilbert spaces," Dynamic Systems and Applications, vol. 17, no. 3-4, pp. 503-513, 2008.

[7] Y. J. Cho, X. Qin, and J. I. Kang, "Convergence theorems based on hybrid methods for generalized equilibrium problems and fixed point problems," Nonlinear Analysis: Theory, Methods E Applications, vol. 71, no. 9, pp. 4203-4214, 2009.

[8] X. Qin, Y. J. Cho, and S. M. Kang, "Convergence theorems of common elements for equilibrium problems and fixed point problems in Banach spaces," Journal of Computational and Applied Mathematics, vol. 225, no. 1, pp. 20-30, 2009.

[9] D. Kinderlehrer and G. Stampacchia, An Introduction to Variational Inequalities and Their Applications, vol. 88 of Pure and Applied Mathematics, Academic Press, New York, NY, USA, 1980.

[10] I. Yamada, "The hybrid steepest descent method for the variational inequality problem over the intersection of fixed point sets of nonexpansive mappings," in Inherently Parallel Algorithms in Feasibility and Optimization and Their Applications (Haifa, 2000), D. Butnariu, Y. Censor, and S. Reich, Eds., vol. 8 of Stud. Comput. Math., pp. 473-504, North-Holland, Amsterdam, The Netherlands, 2001.

[11] L. C. Zeng, Q. H. Ansari, and S. Y. Wu, "Strong convergence theorems of relaxed hybrid steepestdescent methods for variational inequalities," Taiwanese Journal of Mathematics, vol. 10, no. 1, pp. 1329, 2006.

[12] L. C. Zeng, N. C. Wong, and J. C. Yao, “Convergence analysis of modified hybrid steepest-descent methods with variable parameters for variational inequalities," Journal of Optimization Theory and Applications, vol. 132, no. 1, pp. 51-69, 2007.

[13] L.-C. Ceng, H.-K. Xu, and J.-C. Yao, "A hybrid steepest-descent method for variational inequalities in Hilbert spaces," Applicable Analysis, vol. 87, no. 5, pp. 575-589, 2008.

[14] H. K. Xu and T. H. Kim, "Convergence of hybrid steepest-descent methods for variational inequalities," Journal of Optimization Theory and Applications, vol. 119, no. 1, pp. 185-201, 2003.

[15] K. Goebel and W. A. Kirk, Topics in Metric Fixed Point Theory, vol. 28 of Cambridge Studies in Advanced Mathematics, Cambridge University Press, Cambridge, UK, 1990.

[16] P.-E. Maingé, "Approximation methods for common fixed points of nonexpansive mappings in Hilbert spaces," Journal of Mathematical Analysis and Applications, vol. 325, no. 1, pp. 469-479, 2007. 
[17] E. Blum and W. Oettli, "From optimization and variational inequalities to equilibrium problems," The Mathematics Student, vol. 63, no. 1-4, pp. 123-145, 1994.

[18] T. Suzuki, "Strong convergence of Krasnoselskii and Mann's type sequences for one-parameter nonexpansive semigroups without Bochner integrals," Journal of Mathematical Analysis and Applications, vol. 305, no. 1, pp. 227-239, 2005.

[19] V. Colao, G. Marino, and H.-K. Xu, "An iterative method for finding common solutions of equilibrium and fixed point problems," Journal of Mathematical Analysis and Applications, vol. 344, no. 1, pp. 340352, 2008.

[20] S.-S. Chang, H. W. Joseph Lee, and C. K. Chan, "A new method for solving equilibrium problem fixed point problem and variational inequality problem with application to optimization," Nonlinear Analysis: Theory, Methods E Applications, vol. 70, no. 9, pp. 3307-3319, 2009. 